\title{
EXTENSION OF KIRCHHOFF'S FORMULA TO RADIATION FROM MOVING SURFACES
}

F. FARASSAT

M. K. MYERS

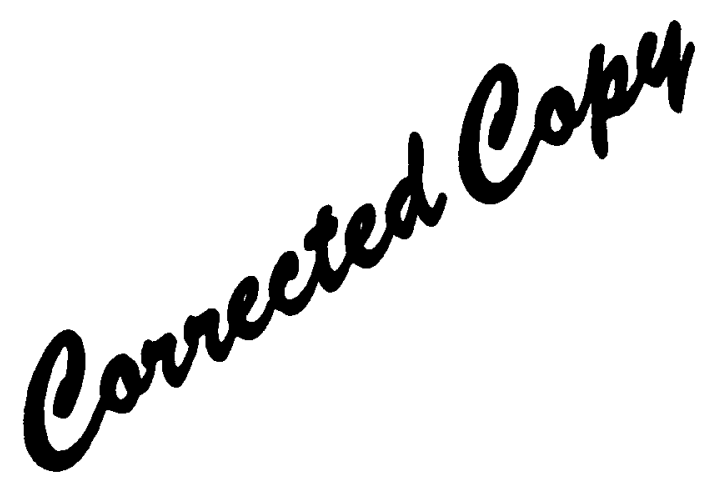

MAY 1987

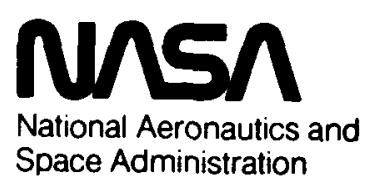

Langley Research Conter

Hampton, Virginia 23665-5225 


\section{ABSTRACT}

Kirchhoff's formula for radiation from a closed surface has been used recently for prediction of the noise of high speed rotors and propellers. Because the closed surface on which the boundary data are prescribed in these cases is in motion, an extension of Kirchhoff's formula to this condition is required. In this paper such a formula, obtained originally by Morgans for the interior problem, is derived for reqions exterior to surfaces moving at speeds below the wave propagation speed by making use of some results of generalized function theory. It is shown that the usual Kirchhoff formula is a special case of the main result of the paper. The general result applies to a deformable surface. However, the special form it assumes for a rigid surface in motion is also noted. Some possible areas of application of the formula to problems of current interest in aeroacoustics are discussed. 


\section{INTRODICTION}

Kirchhoff's formula, published in 1882, is used in the theory of diffraction of liqht and in other electromaqnetic problems [1-2]. It also has many applications to problems of wave propacation in acoustics [3]. One of the novel uses of this formula was proposed by Hawkings for predicting the noise of high speed propellers and helicopter rotors [4]. His idea involves surrounding the rotating blades by a closed surface $S$ which moves with the forward speed of the machine. Inside this surface, nonlinear aerodynamic calculations are carried out which qive the blade loads and the pressure history and its spatial and temporal derivatives on the surrounding surface $s$. In the exterior of this surface, it is proposed that a formula similar to Kirchhoff's be used to calculate propaqation of sound in terms of these surface values. This necessitates extension of the Kirchhoff formula to apply to moving surfaces. In 1930, such an extension was derived for the interior problem by Morgans [5]. His analysis was lenqthy and somewhat complicated, and, in addition, it does not seem to be well known among acousticians. For these reasons, a modern derivation of a generalized Kirchhoff formula applicable to moving surfaces is presented here. It is very likely that specialized versions of this formula have been rederived by many researchers in the context of specific applications. One such case known to the authors is discussed in the last section of the current paper.

In the following section the method used in the derivation of the main result is illustrated by deriving the kirchhoff formula for radiation into the region exterior to a stationary surface $S$. The method involves application of the wave operator to a function which is set equal to zero outside the domain of interest. The function $\Phi$ is thus discontinuous across the surface S. The derivatives in the wave operator are considered as generalized derivatives 
[6-9] which are defined everywhere. The resulting expression then contains terms which involve the jumps in and its derivatives multiplied by Dirac felta functions whose supports are on $S$. These terms can be viewed as source terms for a wave equation which is valid in the entire unbounded space. Kirchhoff's formula is then derived using the Green's function of the wave equation for unbounded space.

In the main section of this paper, the above method is extended to a smooth moving surface. It will be observed that essentially no major difficulties arise in this case. One thus avoids the conventional approach which can become complicated in the case of moving surfaces. The resulting general Kirchhoff formula is then written for piecewise smooth rigid surfaces. It is also shown that the usual formula for a stationary surface is a special case of the qeneral result presented here.

In this paper the wave propagation problem is treated as an acoustic problem and the terminology of acoustics is used. The main result is of course applinahle to many other wave propaqation problems. Immediate applications to the problem of the prediction of the noise of high speed propellers and rotors are expected.

\section{EXAMPLE OF THE MATHEMATICAL APPROACH}

In this section the mathematical method of deriving the main result is illustrated by utilizing it to obtain the kirchhoff formula for a stationary surface $[1-3]$. Consider the closed and bounded smooth surface $S$ described by $f(x)=0$ such that $f>0$ in the exterior of this surface. Assume that $\Phi$ and its first derivatives are continuous in $f>0$ and that $\phi$ satisfies the wave equation

$$
\frac{1}{c^{2}} \frac{\partial^{2} \Phi}{\partial t^{2}}-\nabla^{2} \Phi \equiv \square^{2} \Phi=0
$$


in the exterior of surface $S$, and that $\Phi, \Phi_{t}$ and $\Phi_{n}$ are given on $S$ for $\operatorname{t\varepsilon }(-\infty, \infty)$. Extend $\Phi$ to the interior of $S(f<0)$ by assuming that $\Phi=0$ inside S. To reduce confusion between $\Phi$ and this extended function, the latter function $\tilde{\phi}(\underset{\sim}{x}, t)$ is defined as follows:

$$
\tilde{\Phi}(\underset{\sim}{x}, t)=\left\{\underset{0}{\Phi(x, t)} \quad \begin{array}{lll}
f & >0 & \text { (exterior of } s) \\
& f<0 & \text { (interior of } s \text { ) }
\end{array}\right.
$$

The function $\tilde{\Phi}$ is a discontinuous function whose derivatives do not exist on $f=0$. One can, however, define generalized derivatives for such a function [6-9]. In this paper operators involving generalized derivatives will be denoted hy a har over the operator symbol. It follows from the definition of $\tilde{\Phi}$ that wherever ordinary derivatives of $\tilde{\Phi}$ exist (i.e., interior or exterior to S) one has

$$
\square^{2} \tilde{\Phi}=0
$$

However, one can show, as is done below, that

$$
\tilde{\square}^{2} \tilde{\Phi}=\frac{1}{c^{2}} \frac{\bar{\partial}^{2} \tilde{\Phi}}{\partial t^{2}}-\bar{\nabla}^{2} \tilde{\Phi} \neq 0
$$

It will be seen that what appears on the right side of Ea. (4) leads to the Kirchhoff formula. For the reader who is not familiar with generalized function theory, it is mentioned that one requires considerable conceptual development in going from Eq. (3) to Ea. (4). Examples of applications given in references (7) and [8] mav help in understanding the following steps. 
One can always define the surface $f=0$ in such a way that $|\nabla f|=1$ on this surface. This is done by taking df=dn where dn is distance from the surface $f=0$ along its local normal. With this definition one has $\nabla f=\underset{\sim}{n}$ where $\underset{\sim}{n}$ is the local unit normal pointing into the exterior region. Then, using generalized derivatives [8], one can write at once

$$
\begin{aligned}
& \frac{\bar{\partial} \tilde{\Phi}}{\partial t}=\frac{\partial \tilde{\Phi}}{\partial t}+\tilde{\Phi} \frac{\partial f}{\partial t} \delta(f)=\frac{\partial \tilde{\Phi}}{\partial t}, \\
& \frac{\bar{\partial} 2 \tilde{\Phi}}{\partial t^{2}}=\frac{\partial^{2} \tilde{\Phi}}{\partial t^{2}}, \\
& \bar{\nabla} \tilde{\Phi}=\nabla \tilde{\Phi}+\Phi \underset{\sim}{n} \delta(f), \\
& \bar{\nabla} \tilde{\Phi}=\nabla 2 \tilde{\Phi}+\Phi_{n} \delta(f)+\bar{\nabla} \cdot\left[\Phi_{\sim} \delta(f)\right],
\end{aligned}
$$

where in Fa. (5-c) the function $\phi$ on the riqht side is defined as

$$
\Phi \equiv \lim _{\mathrm{f}+0+} \Phi(\underset{\sim}{x}, t)
$$

The function ${ }_{n}$ in Ea. $(5-d)$ is the normal derivative of $\Phi$, and is defined similarly. The Dirac delta function is denoted $\delta(f)$. From Eq. (5) one obtains

$$
\bar{\square}^{2} \tilde{\Phi}=\square^{2} \tilde{\Phi}-\Phi_{n} \delta(E)-\bar{\nabla} \cdot\left[\Phi_{\sim} \delta(E)\right]=-\Phi_{n} \delta(E)-\bar{\nabla} \cdot\left[\Phi_{\sim} \delta(E)\right] .
$$

Here, Eq. (3) is used in the last step. This completes the determination of the right side of Ea. (4).

Equation (7) is valid in the entire unbounded space. One can now use the Green's Function for the wave equation in unbounded space, $\delta(g) / 4 \pi r$, where 


\section{ORIGINAL PAGE IS}

OF POSP QUALITY

$q=\tau-t+r / c$ and $r=|\underset{\sim}{x}-y|$. The space-time variables of the observer and source are denoted $(\underset{\sim}{x}, t)$ and $(x, \tau)$, respectively. The speed of sound is $c$. Eguation (7) then yields

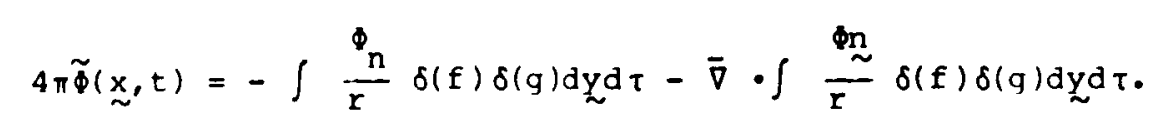

The space inteqrals in Eq. (8) are over the entire unbounded space and the time integral is over $(-\infty, t)$. The divergence operator on the right acts on the variable $x$. Ise the fact that the volume element dy $=d f d S$, where dS is the element of area of the surface $s: f=0$. Then let $\tau+q$ noting that the Jacobian of the transformation $|\partial \tau / \partial g|=|\partial g / \partial \tau|^{-1}$ is unity. Integration over $f$, by virtue of the delta function $\delta(f)$, restricts the expressions to $f=0$. Suhsequent inteqration over 9 restricts the expressions to $q=0$, which introduces the retarded time. Equation ( 8 ) thus becomes

$$
4 \pi \tilde{\Phi}(\underset{\sim}{x}, t)=-\int_{f=0} \frac{\left[\Phi_{n}\right]}{r} d S-\bar{\nabla} \cdot \int_{f=0} \frac{[\Phi]}{r} \underset{\sim}{\sim} d S
$$

Here the square bracket stands for evaluation of the functions at the retarded time; i.e., $[\phi] \equiv \phi(\underset{\sim}{y}, t-r / c)$.

Taking the gradient operator inside the second integral of Eq. (9) and collecting terms of $O(1 / r)$ and $O\left(1 / r^{2}\right)$, one gets

$$
\begin{aligned}
& 4 \pi \tilde{\phi}(\underset{\sim}{x}, t)= \begin{cases}4 \pi \Phi(\underset{\sim}{x}, t) & f>0 \\
0 & f<0\end{cases} \\
& =\int_{f=0} \frac{\left[c^{-1} \Phi \tau \cos \theta-\Phi_{n}\right]}{r} d S+\int_{f=0} \frac{[\Phi] \cos \theta}{r^{2}} d S
\end{aligned}
$$


Here $\Phi_{\tau}=\partial \Phi(\chi, \tau) / \partial \tau$ and $\theta$ is the angle between $\mathrm{n}$ (pointing into the exterior region) and the radiation direction $\underset{\sim}{r}=\underset{\sim}{x}-\underset{\sim}{*}$. For the exterior region $f>0$, this is Kirchhoff's formula for a stationary surface $S$. The present method automatically shows that for the observer inside the surface $s$ the inteqrals in Kirchhoff's formula yield a zero (null) field. This means that $\Phi_{\text {, }} \Phi_{n}$ and $\Phi_{\tau}$ are not independent on the surface S. Equation (10) is an identity which may he utilized in various ways. As it stands it may be considered as an integral representation of $\Phi$ at points exterior to $S$ in terms of surface quantities. Another interpretation follows if the observer point $\underset{\sim}{x}$ is taken on $s$ itself; in that case, Eq. (10) becomes an inteqral equation governing $\Phi$ on S. This latter interpretation is the basis of modern boundary element methods. It is seen that the integrals of $\mathrm{Eq} \cdot(10)$ remain valid for a piecewise smooth surface $\mathrm{S}$. It is noted that in the usual applications of Eq. (10) $\Phi$ is taken as a complex harmonic function of time so that the temporal part of $\phi$ disappears in Ea. (10); the resulting Kirchhoff formula pertains to the complex amplitude of $\Phi[1-3]$. The amplitude satisfies Helmholtz' equation. In this case one could hegin the above procedure from a Helmholtz equation rather than from the wave equation as is done above.

THE GENERAL KIRCHHOFF FORMULA

In this section, the Kirchhoff formula for moving surfaces will be derived. Let $f(\underset{\sim}{x}, t)=0$ describe the moving surface $s$ which is assumed to be piecewise smooth. The function $f$ is defined as before such that $f>0$ outside $S$ and $\nabla f$ $=\underset{\sim}{\mathrm{o}}$ on $\mathrm{f}=0$. The function $\Phi$ and its first derivatives are assumed to he continuous and $\phi$ satisfies Ea. (1). This function is again extended to $\tilde{\Phi}$ by 


\section{ORIGINAL PAGE IS \\ OF POOR QUALITY}

FA. (2) so that Eq. (3) is satisfied both inside and outside S. The terms on the right side of Ea. (4) are found by the same technique as presented above. Taking the generalized derivative of $\tilde{\Phi}$ with respect to time gives

$$
\frac{\bar{\partial} \tilde{\phi}}{\partial t}=\frac{\partial \tilde{\Phi}}{\partial t}+\Phi \cdot \frac{\partial f}{\partial t} \delta(\mathrm{f})=\frac{\partial \tilde{\Phi}}{\partial t}-\Phi v_{n} \delta(\mathrm{f})
$$

where $v_{n}=-\partial f / \partial t$ is the local normal velocity of $s$ with respect to the undisturhed medium. Differentiating both sides of Eq. (11) once more yields

$$
\frac{\bar{\partial} 2 \tilde{\Phi}}{\partial t^{2}}=\frac{\partial^{2} \tilde{\Phi}}{\partial t^{2}}-\Phi_{t(x)} v_{n} \delta(E)-\frac{\bar{\partial}}{\partial t}\left[\Phi v_{n} \delta(E)\right]
$$

where $\Phi_{t(x)}=\partial \Phi /\left.\partial t\right|_{x}$. The generalized Laplacian of $\Phi$ is given by Eq. (5-d) as before. From this and Eq. (12), one obtains

$$
\bar{\square}^{2} \tilde{\phi}=-\left(\Phi_{n}+\frac{1}{c} M_{n} \Phi_{t(x)}\right) \delta(f)-\frac{1}{c} \frac{\bar{\partial}}{\partial t}\left[M_{n} \Phi \delta(f)\right]-\bar{\nabla} \cdot[\Phi \underset{\sim}{n} \delta(f)]
$$

where $M_{n}=v_{n} / c$ is the local normal Mach number on $s$. In the following, partial derivatives with respect to time holding different sets of space variables fixed will appear. The notation introduced above $\left(e \cdot g \cdot \Phi_{t(x)}\right)$ will be maintained wherever necessary in order to prevent confusion. The source terms in Eq. (13) can be written in other forms by performing the temporal and spatial derivatives in the second and the third terms, respectively. The above form, however, seems to require the fewest algebraic manipulations in the following analysis.

Ipon use of the Green's Function of the wave operator in unbounded space, Eq. (13) qivies 
$4 \pi \tilde{\phi}(\underset{\sim}{x}, t)=-\int \frac{1}{r}\left(\phi_{n}+\frac{1}{c} M_{n} \Phi_{\tau(y)}\right) \delta(f) \delta(q) d \underset{\sim}{d} \tau-\frac{1}{c} \frac{\bar{\partial}}{\partial t} \int \frac{1}{r} M_{n} \Phi \delta(f) \delta(g) d \underset{\sim}{\partial} d \tau$

$$
-\bar{\nabla} \cdot \int \frac{1}{r} \operatorname{n}_{\sim} \delta(f) \delta(q) d \chi d \tau
$$

where $\Phi_{\tau(y)}=\partial \Phi(\chi, \tau) / \partial \tau$.

If the spatial variables $\underset{\sim}{y}$ are transformed to Lagrangian variables $\underset{\sim}{\eta}$ and if the transformation $\tau * g$ is employed and the delta functions integrated out, then Eq. (14) can immediately be written in the form of Eq. (5.3) of Reference [10]. Such an approach leaves the divergence and the time derivatinve operators outside the inteqrals, however, which renders the resulting formula unsuitable for numerical application. In the current paper Eq. (14) will be manipulated differently to lead, as will be seen, to a more practically useful resist.

Before interpreting the inteqrals in Eq. (14), the following relation is used to convert the space derivatives in the last integral to a time derivative:

$$
\frac{\bar{\partial}}{\partial x_{i}}\left[\frac{\delta(g)}{r}-\right]=-\frac{1}{c} \frac{\bar{\partial}}{\partial t}\left[\frac{\hat{r}_{i} \delta(g)}{r}\right]-\frac{\hat{r}_{i} \delta(g)}{r^{2}}
$$

where $\underset{\sim}{\vec{r}}=(\underset{\sim}{x-y}) / x$ is the unit radiation vector. Note that in this relation the somrce variables $(\underline{\sim}, \tau)$ are held fixed. Equation (14) can now be written as

$$
\begin{aligned}
4 \pi \widetilde{\Phi}(\underset{\sim}{x}, t) & =-\int \frac{1}{r}\left(\Phi_{n}+\frac{1}{c} M_{n} \Phi_{\tau(y)}\right) \delta(f) \delta(q) d \underset{\nu}{d \tau}+\int \frac{1}{r^{2}} \Phi \cos \theta \delta(f) \delta(g) d y d \tau \\
& +\frac{1}{c} \frac{\bar{\partial}}{\partial t} \int \frac{1}{r}\left(\cos \theta-M_{n}\right) \Phi \delta(f) \delta(q) d \underset{\sim}{y} d \tau
\end{aligned}
$$


There are two types of inteqrals in Eq. (16), which will be denoted

$$
\begin{aligned}
& I_{1}=\int \Omega_{1} \delta(f) \delta(q) d y \sim d \\
& I_{2}=\frac{1}{c} \frac{\bar{\partial}}{\partial t} \int Q_{2} \delta(f) \delta(q) d \underset{\sim}{d \tau}
\end{aligned}
$$

The technique of evaluation of these integrals will be qiven below.

Although in many applications of the Kirchhoff formula in acoustics the surface $S$ is taken as riqid, for completeness $S$ is here assumed to be deformahle. Let $s$ he described by the surface coordinates $\left(u^{1}, u^{2}\right)$ and assume $\mathrm{u}^{3}=\mathrm{f}$. This mapping is only required in the vicinity of $\mathrm{f}=0$. Let the mapping $\left(u^{1}, u^{2}, u^{3}\right)+\underset{\sim}{y}$ be a differentiable function of time $\tau$. Denote the determinant of the coefficients of the first fundamental form on $s$ by $g_{(2)}=g_{11} g_{22}-$ $q_{12}{ }^{2}$, where the $g_{i j}(i, j=1,2)$ are the metric tensor components. Then $g_{(2)}$ is a function of time $\tau$ in addition to $\left(u^{1}, u^{2}\right)$. For example, consider a sphere of radius $R(\tau)$ whose center is moving at speed $\underset{\sim}{U}(\tau)$. Let $\left(u^{1}, u^{2}\right)$ be the spherical surface coordinates on this sphere as shown in Fig. 1 . Then if at $\tau=0$ the

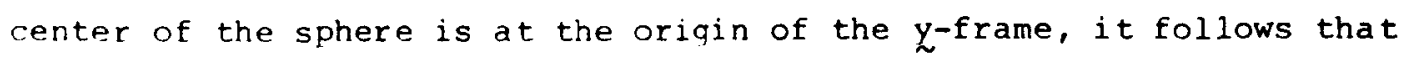

$$
\begin{aligned}
& y_{1}=y_{1}^{0}(\tau)+\left[u^{3}+R(\tau)\right] \cos u^{1} \sin u^{2} \\
& y_{2}=y_{2}^{0}(\tau)+\left[u^{3}+R(\tau)\right] \sin u^{1} \sin u^{2} \\
& y_{3}=y_{3}^{0}(\tau)+\left[u^{3}+R(\tau)\right] \cos u^{2}
\end{aligned}
$$

where

$$
y^{0}(\tau)=\int_{0}^{\tau} \underset{\sim}{J}\left(\tau^{\prime}\right) d \tau^{\prime}
$$


Also, it is seen that on $S$

$$
\sqrt{q}(2)=R^{2}(\tau) \text { sin } u^{2}
$$

To evaluate $I_{1}$, the following successive transformations are carried out: $\chi+\left(u^{1}, u^{2}, u^{3}\right)$ and then $\tau \rightarrow q$. The Jacobians of transformations are 1 and $1,\left(1-M_{r}\right)$, respectively, where $M_{r}=\underset{\sim}{v} \cdot \hat{r} / c$. Here $\underset{\sim}{v}=\partial y / \partial \tau$ holding $\left(u^{1}, u^{2}, u^{3}\right)$ fixed. After the first transformation and integration with respect to $f(i . e$. $\left.u^{3}\right), I_{1}$ is given by

$$
I_{1}=\int_{-\infty}^{t} \int_{D(S)} \rho_{1}\left(u^{1}, u^{2}, 0, \tau\right) \delta(g) \sqrt{g(2)} d u{ }^{l} d u{ }^{2} d \tau
$$

where $D(S)$ is the domain of $S$ in $\left(u^{1}, u^{2}\right)$-space. Note that even if $s$ is deformable, the limits of the inner integrals can be made time independent and are assumed so here (see Eiq. 1). The deformation of $s$ appears in the variation of $9(2)$ with $\tau$. The second transformation $\tau \rightarrow g$ and the subsequent integration with respect to $g$ restrict the integrand to $q=0$ and yield

$$
\left.I_{1}=\int_{D(S)}\left[\frac{Q_{1} \sqrt{q}(2)}{1-M_{r}}\right]\right]_{q=0} d u l_{d u}^{2}
$$

The interpretation of the integrand is as follows. If the relation $g=0$ is explicitly written (remembering that $u^{3}=f=0$ also in the integrand)

$$
g=\tau-t+\left|\underset{\sim}{x}-\underset{\sim}{y}\left(u^{2}, u^{2}, 0, \tau\right)\right| / c=0
$$

then the solution in source time, $\tau^{\star}$, of this equation is the emission time which must be used in the integrand of Fa. (23). Note that since $|\underset{\sim}{v}|<c$ by 


\section{ORIGINAL PAGE IS \\ OF. POOR QUALITY.}

assumption, Ea. (24) has a unique solution. Equation (23) will thus be written as

$$
I_{1}=\int_{D(S)}\left[\frac{Q_{1} \sqrt{q}(2)}{1-M_{r}}\right] \tau^{\star} d u^{1} d u^{2}
$$

Since $D(S)$ is independent of $t, I_{2}$ follows immediately from Eg. (18) as

$$
I_{2}=\frac{1}{C} \int_{D(S)} \frac{\partial}{\partial t}\left[\frac{Q_{2}^{\sqrt{q}(2)}}{1-M_{r}} \tau_{\tau^{*}} d u^{1} d^{2} u^{2}=\frac{1}{C} \int_{D(S)}\left[\frac{1}{1-M_{r}}-\frac{\partial}{\partial \tau}\left\{\frac{P_{2} \sqrt{g(2)}}{1-M_{r}}\right\}\right] \tau^{\star} d u^{l} d u^{2}\right.
$$

where the relation $\partial / \partial t=\left(1-M_{Y}\right)^{-1} \partial /\left.\partial \tau\right|_{\underset{\sim}{u}}$, obtained from Eq. $(24)$, is used in the last expression. Note carefully that in taking $\partial / \partial \tau$ the variables $u^{1}$ and $u^{2}$ are kept fixed.

After usinq the results of Eqs. (25) and (26), Eq. (16) can be written as

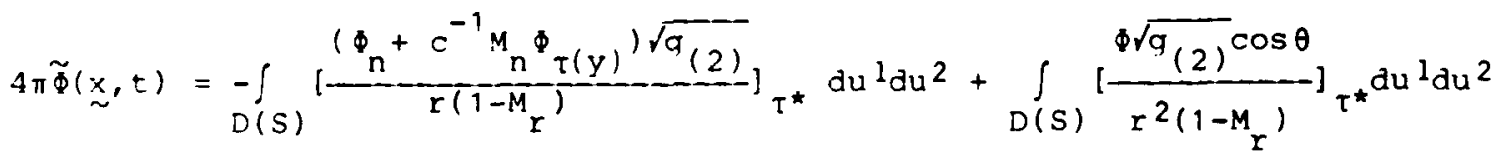

$$
\begin{aligned}
& +\frac{1}{C} \int_{D(S)}\left\{\frac{1}{1-M_{r}}-\frac{\partial}{\partial \tau}\left\{-\frac{\left(\cos \theta-M_{n}\right) \Phi \sqrt{g}(2)}{r\left(1-M_{r}\right)}\right\}\right\}_{\tau} d u^{2} d u^{2}
\end{aligned}
$$

This result is equivalent to that of Morqans [5] and is referred to here as the igeneral kirchhoff formula. The notation of the present paper, however, differs from Morgans', who addressed the interior problem.

There is a somewhat subtle point which should be made, however, about the result qiven in Reference [5]. The quantity $[\sqrt{g(2)}] \tau^{\star} d u^{1} d u^{2}$ is there written ds, but this is not the element of area of the physical surface $S$ which is defined by $f i x i n q t$ in $f(y, \tau)=0$. That element is $\sqrt{q(2)}$ du ${ }^{1} d u^{2}$. The difference arises hecause as $s$ deforms in space any element specified by $\left(u^{1}, u^{2}\right)$ has dif- 
ferent emission times $\tau^{\star}$ for a fixed $\underset{\sim}{x}$ and $t$. Corresponding to each $\tau^{\star}$ the surface $S$ has a different shape. Although the main result of Morgans is correct if interpreted as in the present paper, in some of his elementary examples there is an ambiguity in the meaning of dS. It should be noted that Morgans' result (27) is claimed to be erroneous by the authors of Reference [10]. However, they do not specifically point out the nature of the error. The modern derivation qiven above indicates that Morgans' formula is, in fact, correct, $z$ conclusion which was made also by Munro [11].

Finally, it must be mentioned that the moving surface $S$ in the above analysis can be unbounded provided that it divides the space into interior and exterior parts. An open bounded or unbounded surface can be considered as the limiting case of a closed surface of infinitely small thickness to which the Kirchhoff formula can be applied.

If $S$ is stationary, then $M_{n}$ and $M_{r}$ are zero and $q(2)$ is time independent. Also $\tau^{\star}$ is simply t-r/c. Inder these conditions, Eq. (27), reduces to the Kirchhotf formula for stationary surfaces, Eq. (10).

\section{ALTERNATE FORM FOR APPLICATIONS}

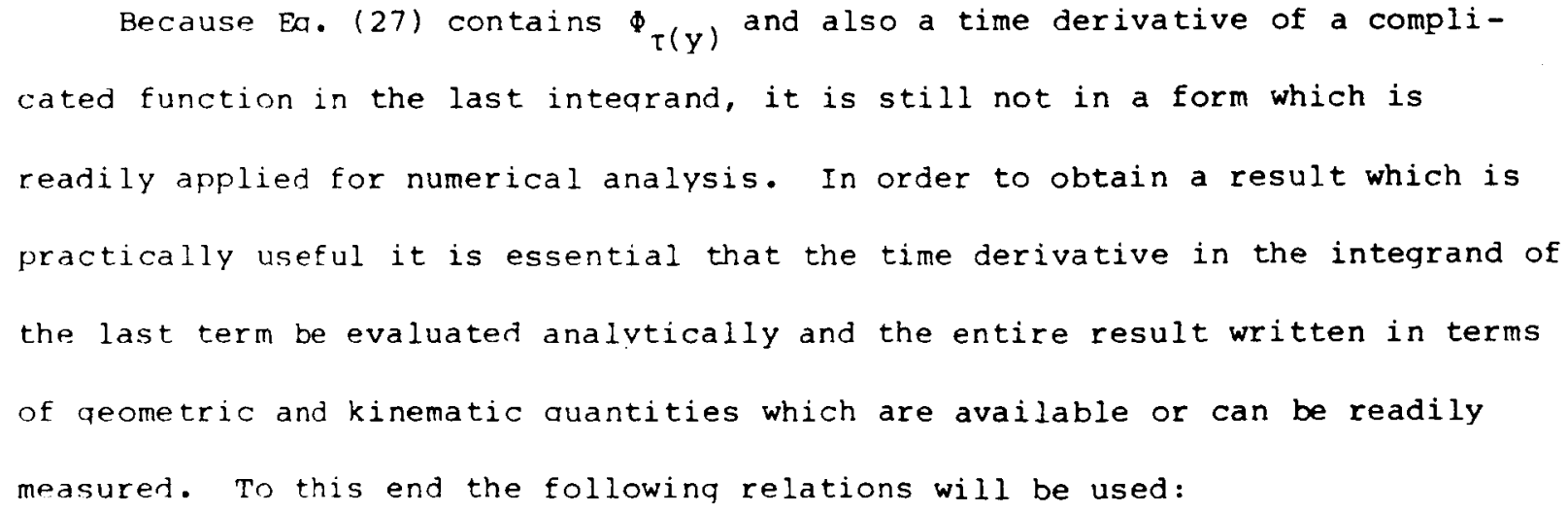




$$
\begin{aligned}
& \frac{1}{c} \frac{\partial M_{r}}{\partial \tau}=\frac{1}{c} \underset{\sim}{\dot{M}} \cdot \underset{\sim}{\sim}+\frac{1}{r}\left(M_{r}^{2}-M^{2}\right) \\
& \frac{1}{c} \frac{\partial \cos \theta}{\partial \tau}=\frac{1}{c} \dot{\sim} \cdot \underset{\sim}{r}+\frac{1}{r}\left(M_{r} \cos \theta-M_{n}\right) \\
& \frac{1}{c} \frac{\partial M_{n}}{\partial \tau}=\frac{1}{c}(\underset{\sim}{M} \cdot \underset{\sim}{n}+\underset{\sim}{M} \cdot \underset{\sim}{\dot{n}})
\end{aligned}
$$

where the dot over $\underset{\sim}{M}$ and $\underset{\sim}{\sim}$ denote source time derivative keeping $\left(u^{1}, u^{2}\right)$ fixed. In adition, the following definitions are introduced:

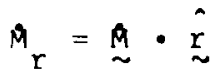

$$
\begin{aligned}
& \dot{n}_{r}=\underset{\sim}{\dot{n}} \cdot \hat{\sim} \\
& m_{n}=\underset{\sim}{n} \cdot \underset{\sim}{n} \\
& \dot{n}_{M}=\dot{n} \cdot M \\
& \frac{1}{\sqrt{q}(2)} \frac{\partial \sqrt{g}(2)}{\partial \tau}=\dot{\sigma}
\end{aligned}
$$

Note that in the last inteqral of Eq. (27), a new time rate of chanqe of $\Phi$ will appear when $\left(u^{1}, u^{2}\right)$ is kept fixed. This is denoted $\$$. The relation between $\Phi_{\tau(y)}$ and $\dot{\phi}$ is obtained as follows:

$$
\begin{aligned}
\dot{\Phi} & =\left\{\frac{\partial}{\partial \tau} \Phi[\underset{\sim}{\underline{u}}(\underset{\sim}{u}, \tau), \tau]\right\}_{u_{3}=0}=\left\{\frac{\partial \Phi}{\partial y_{i}} \frac{\partial y_{i}}{\partial \tau}+\frac{\partial \Phi}{\partial \tau}\right\}_{u_{3}}=0 \\
& =\left\{v_{\sim}^{v} \cdot \nabla \Phi+\Phi_{\tau(y)}\right\}_{u_{3}=0}=c M_{n} \phi_{n}+c M_{\tau} \cdot \nabla_{2} \Phi+\Phi_{\tau(y)}
\end{aligned}
$$


where $\underset{\sim}{u}=\left(u^{1}, u^{2}, u^{3}\right), \underset{\sim}{v}=\partial y /\left.\partial \tau\right|_{\underset{\sim}{u}}$ and ${\underset{\sim}{M}}_{t}$ is the tangential component of the Mach number vector $\underset{\sim}{M}=\underset{\sim}{v} / C$ on $S$. Also $\nabla_{2}$ stands for the surface gradient operator.

Equation (27) can now be written as

$$
4 \pi \tilde{\Phi}(x, t)=\int_{D(S)}\left[\frac{E_{1} \sqrt{q}(2)}{r\left(1-M_{r}\right)}\right] \tau^{\star} d u^{1} d u^{2}+\int_{D(S)}\left[\frac{\Phi E_{2} \sqrt{q(2)}}{r^{2}\left(1-M_{r}\right)}\right] \tau^{\star} d u^{1} d u^{2}
$$

whers the expressions $E_{1}$ and $E_{2}$ are

$$
\begin{aligned}
E_{1}=\left(M_{n}^{2}-1\right) \Phi_{n}+M_{n \sim t} M_{2}-\nabla_{2}-c^{-1} M_{n} \dot{\phi}+\frac{c^{-1}}{1-M_{r}}\left[\left(\dot{n}_{r}-\dot{M}_{n}-\dot{n}_{M}\right) \Phi\right. \\
+\left(\cos \theta-M_{n}\right) \dot{\Phi}+\left(\cos \theta-M_{n}\right) \dot{\phi} \dot{0}+\frac{c^{-1}}{\left(1-M_{r}\right)^{2}}\left[\dot{M}_{r}\left(\cos \theta-M_{n}\right) \phi\right] ; \\
E_{2}=\cos \theta+\frac{1}{1-M_{r}}-\left[2 M_{r} \cos \theta-M_{n} M_{r}-M_{n}\right]+\frac{1}{\left(1-M_{r}\right)^{2}}\left[\left(\cos \theta-M_{n}\right)\left(M_{r}^{2}-M^{2}\right)\right]
\end{aligned}
$$

If the surface $S$ is assumed rigid, then $q_{(2)}$ is independent of $T$ and

$$
\dot{\sim}=\underset{\sim}{\omega} \times \underset{\sim}{n}
$$

where $\underset{\sim}{\omega}$ is the anqular velocity of $s$. One can now write $d s=\sqrt{9(2)} d u^{1} d^{2}$ for the element of area of $\mathrm{s}$. Equation ( 31 ) can thus be written for a rigid surface as

$$
4 \pi \tilde{\Phi}(\underset{\sim}{x}, t)=\int_{S}\left[\frac{E_{1}}{r\left(1-M_{r}\right)}\right] \tau^{*} d S+\int_{S}\left[\frac{\Phi E_{2}}{r^{2}\left(1-M_{r}\right)}\right] \tau^{*} d S
$$

where $E_{1}$ and $E_{2}$ are aqain given by Eq. (32) using Eq. (33) where needed. The 


\section{ORIGINAL PAGE IS \\ OF POOR QUALITY}

general Kirchhoff formula of Eqs. (31) and (34) is now in a form which is suitable for practical applications. These results appear not to have been published elsewhere. Once more it can be checked that the Kirchhoff formula for stationary surfaces, Eq. (10), can be obtained from these equations. Again it can be seen that both Eqs. (31) and (34) remain valid for a piecewise smooth surface $S$.

REMARKS ON EXTENSION TO SUJPERSONIC MOTION

A more qeneral Kirchhoff formula can he derived which is valid for deformable, piecewise smooth surfaces in arbitrary motion (subsonic or supersonic). The derivation of this result is very lengthy and complicated, and it will not be written out here. It relies on the solution of the following wave equation qiven by the authors [12]:

$$
\bar{\square}^{2} \Phi=\bar{\nabla}_{4} \cdot[Q|\nabla f| \delta(\mathrm{f})]
$$

In this equation $\nabla_{4}=\left(\nabla, c^{-1} \partial / \partial t\right)$ and $Q$ is a 4 -vector. Note that $|\nabla f|=1$ in the present paper. Equation (13) can be written as

$$
\bar{\square}^{2 \tilde{\Phi}}=-\left(\Phi_{n}+\frac{1}{c} M_{n} \Phi_{t(x)}\right) \delta(E)-\bar{\nabla}_{4} \cdot[\ell \delta(E)]
$$

where $\varrho=\Phi\left(n_{\sim}, M_{n}\right)$. Now the Green's function of the wave equation can be applied to find $\tilde{\Phi}$ utilizing the result of reference [12] for the second term of Eg. (36). The resulting Kirchhoff formula is more general than the formula presented here. However, the various forms of the general Kirchhoff formula of the current paper appear to be adequate for present applications in acoustics. 


\section{APPLICATIONS IN ACOUSTICS}

As mentioned in the introduction, application of the Kirchhoff formula has been proposed for rotor noise prediction. Attempts have also been made by Forsyth and Korkan [13] to use this formula for high speed propeller noise calculation. These authors have proposed using the Kirchhoff formula for a stationary surface which, as shown above, is very different from the general

Kirchhoff formula. The surface $S$ in the work of Forsyth and Korkan is taken as a finite cylinder, fixed with respect to the propeller, with its axis along that of the propeller. This surface is therefore in motion with respect to the unbounded acoustic medium, and the general Kirchhoff formula must be applied to $S$.

One of the interesting applications of the general kirchhoff formula may be in the prediction of the noise of high bypass ratio turbofans. These propulsors are currently favored in aircraft designs because of their high efficiency. By using a cylindrical surface $S$ with its axis along the fan axis and its ends at the fan inlet and exhaust, Fiq. 2, the main result of the present paper can be applied to predict the radiation pattern. There are several sophisticated turbomachinery aerodynamics codes which can supply the needed input data to the Kirchhoff formula.

Lyrintzis and Georqe have developed a specialized Kirchhoff formula to calculate the far field noise from a single helicopter blade interacting with a vortex [14]. Their formula applies to uniform motion of a rigid surface and is derived by using Green's theorem. It is equivalent, in their case, to the qeneral Kirchhoff formula derived here, Eq. (34). 


\section{ORIGINAL PAGE IS \\ OF POOR QUALITY.}

ACKNOWL EDGMENTS

The authors are indebted to Prof. H. S. Ribner for numerous suggestions which qreatly improved the presentation of the foregoing results. Thanks are

also due to prof. A. R. Georqe for his useful comments. The work of the second author was supported by NASA under Cooperative Agreement NCC1-14.

\section{REFERENCES}

1. D. S. Jones 1964 The Theory of Electromagnetism. Oxford, Pergamon Press.

2. Julius Adams Stratton 1941 Electromagnetic Theory. New York, McGraw-Hil1 Book Company.

3. Allan D. Pierce 1981 Acoustics - An Introduction to Its Physical Principles and Applications. New York, McGraw-Hill Book Company.

4. D. L. Hawkings 1979 westland Research Paper 599. Noise Generation by Transonic Open Rotors.

5. W. R. Morqans 1930 Philosophical Maqazine, 9(S. 7, No. 55), 141-161. The Kirchhoff Formula Extended to a Moving Surface.

6. I. M. Gel'fand and G. E. Shilov 1964 Generalized Functions, Volume 1, Properties and Operations. New York, Academic Press.

7. R. P. Kanwal 1983 Generalized Functions - Theory and Technique. New York, Academic Press.

8. F. Farassat 1977 Journal of Sound and Vibration, 55, 165-193. Discontinuities in Aerodynamics and Aeroacoustics: The Concept and Applications of Generalized Derivatives.

9. D. S. Jones 1966 Generalized Functions. London, McGraw-Hill Book Company. 10. J. E. Ffowcs Williams and D. L. Hawkings 1969 Philosophical Transactions of the Royal Society, 264A, 321-342. Sound Generation by Turbulence and Surfaces in Arbitrary Motion. 
11. D. H. Munro $1980 \mathrm{Ph} \cdot \mathrm{D}$. Dissertation, Massachusetts Institute of Technoloay. The Production of Sound by Moving Objects.

12. F. Farassat and M. K. Myers 1986 First IMACS International Symposium on Computational Acoustics, Yale Iniversity, August 6-8. The Moving Boundary Problem for the Wave Equation: Theory and Application.

13. D. W. Forsyth and K. D. Korkan 1987 AIAA-87-0254, AIAA 25th Aerospace Sciences Meeting, January 12-15, Reno, Nevada. Computational Aeroacoustics of Propeller Noise in the Near and Far Field.

14. A. S. Lyrintzis and A. R. George 1987 American Helicopter Society National Sperialists' Meeting on Aerodynamics and Aeroacoustics, February 25-27, Arlington, Texas. Transonic Blade-Vortex Interactions: The Far Field. 
ORIGINAL PAGE IS

OE POOR QUALITY.
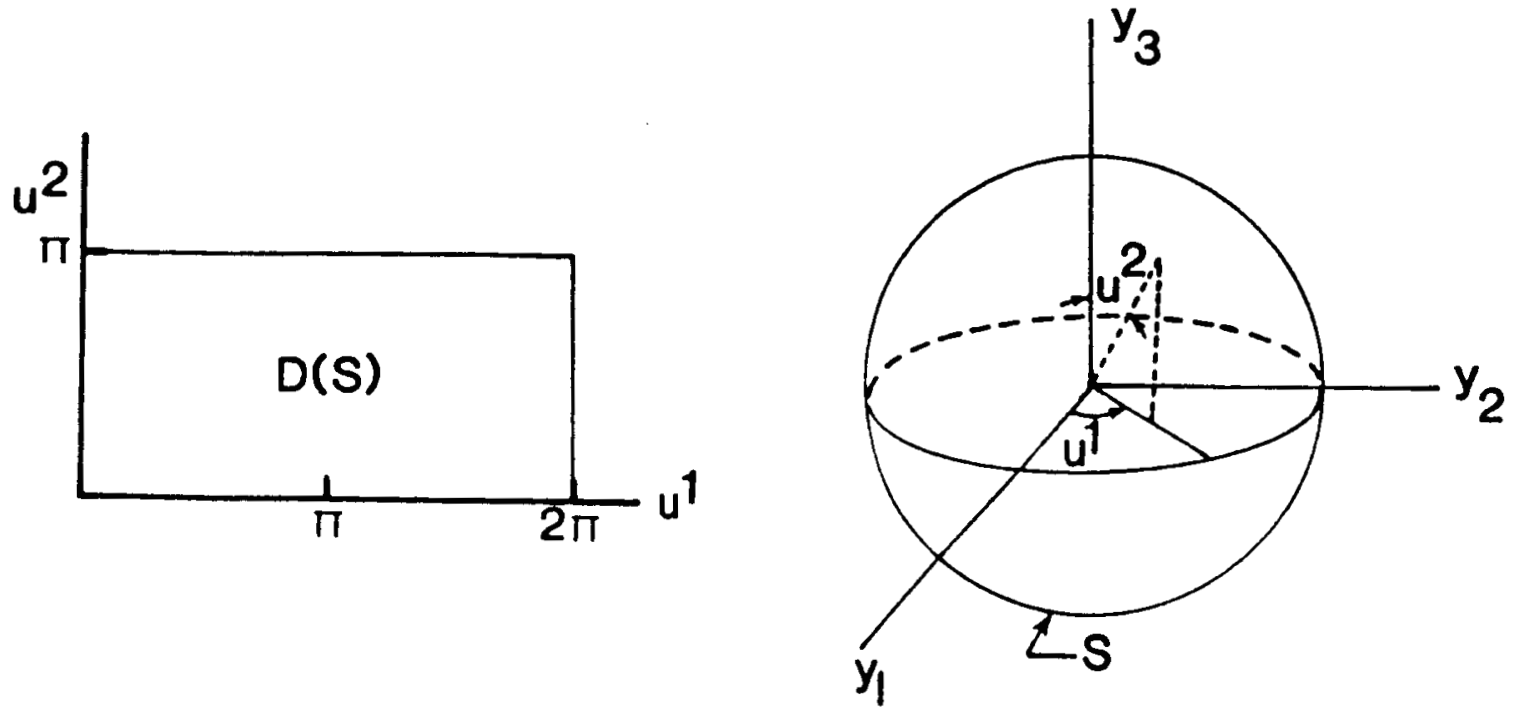

Figure 1: The mapping $y \rightarrow\left(u^{1}, u^{2}\right)$ Doma in $O(S)$ is independent of time. 


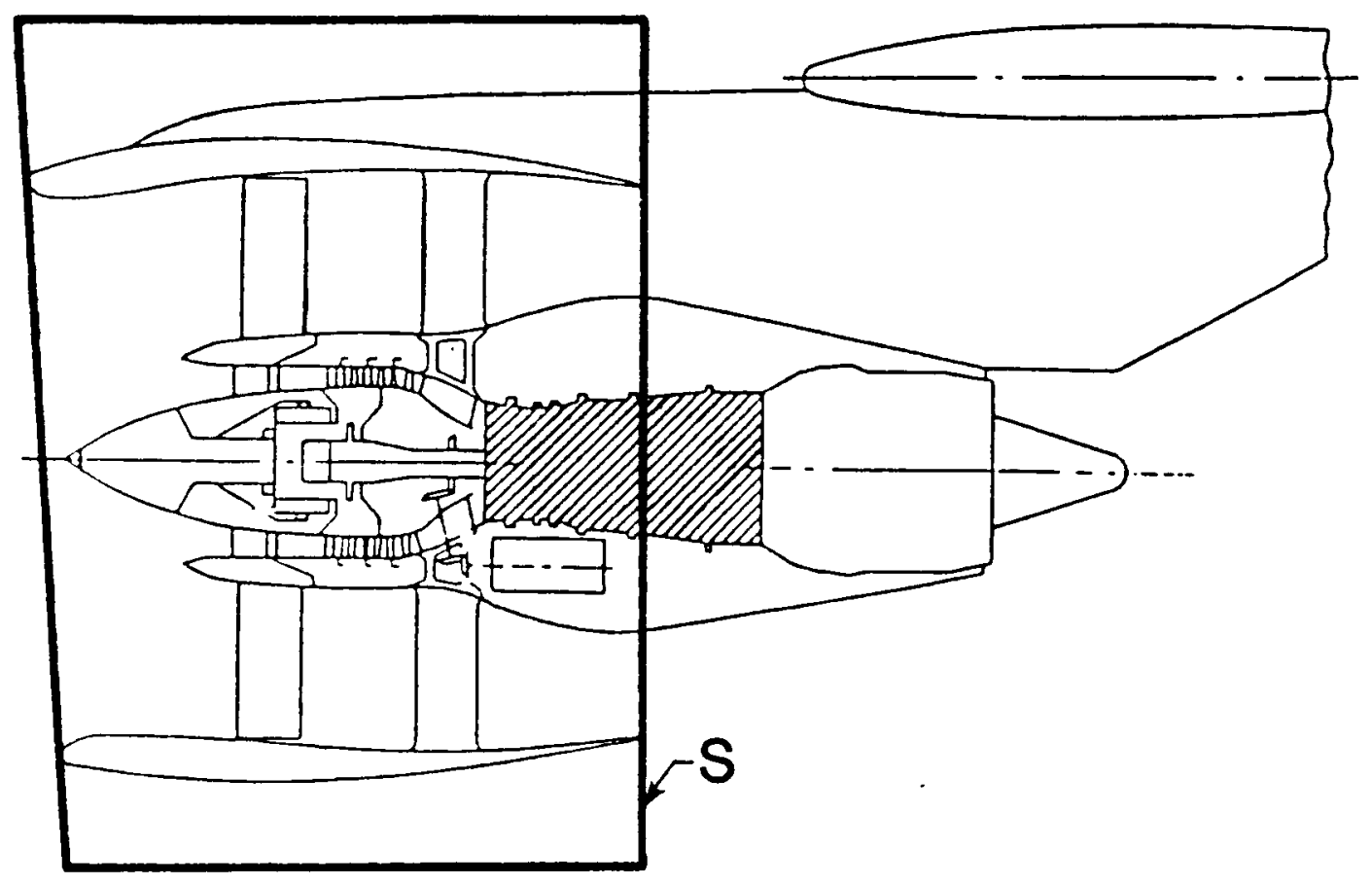

Figure 2: Illustrating a surface $S$ appropriate for turbofan noise analys is.

ORIGINAL PAGE IS OE ROOR QUALITY. 


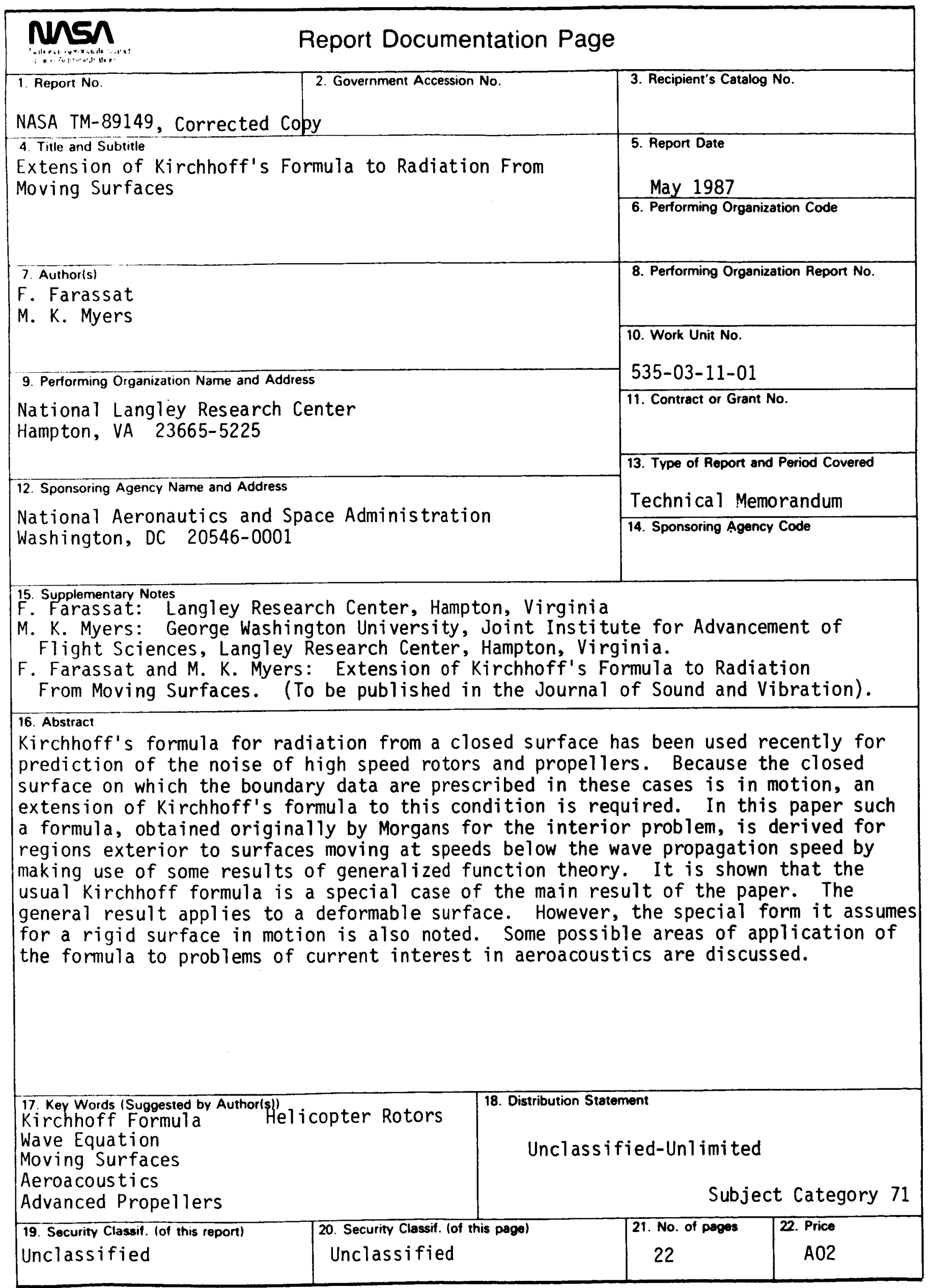

